\title{
Horizontal transfer of the immunoglobulin A1 protease gene (iga) from Streptococcus to Gemella haemolysans
}

\author{
Nobuko Takenouchi-Ohkubo, ${ }^{1,2}$ Lotte M. Mortensen, ${ }^{1}$ Kim R. Drasbek, ${ }^{1}$ \\ Mogens Kilian ${ }^{1}$ and Knud Poulsen ${ }^{1}$ \\ ${ }^{1}$ Institute of Medical Microbiology and Immunology, The Bartholin Building, University of \\ Aarhus, DK-8000 Aarhus C, Denmark \\ ${ }^{2}$ Department of Pathology, Nihon University School of Dentistry, Tokyo, Japan
}

\begin{abstract}
Bacterial $\lg \mathrm{A} 1$ proteases share the ability to cleave human $\lg \mathrm{A} 1$ at the hinge region. Nature has developed this trait along at least five independent evolutionary lineages. To obtain further insight into the phylogeny and function of IgA1 proteases, the nucleotide sequence of the iga gene that encodes the IgA1 protease was determined from two Streptococcus mitis strains and one Gemella haemolysans strain. Heterologous expression in Escherichia coli confirmed that the genes encode human IgA1-cleaving activity. IgA1 proteases from Streptococcus and G. haemolysans shared structural features, including a motif typical for zinc-dependent metalloproteases of clan MA(E) family M26 and an N-terminal signal sequence followed by an LPXTG cell-wall-anchor motif and two putative membrane-spanning domains. In addition, they all harboured a repeat region preceding the active site of the protease. In the streptococcal IgA1 proteases, a G5 domain, which has been suggested to bind $N$-acetylglucosamine, was identified. Conservation of these structures in otherwise diverse proteases suggests that they are essential to the biological function of the enzyme. The phylogenetic distribution of homologous iga genes and conservation of gene order in the iga gene region in different Streptococcus species, combined with the sequence homologies, strongly suggest that the iga gene is more ancient in Streptococcus than in G. haemolysans, and therefore that the $\lg A 1$ protease gene was transferred from Streptococcus to G. haemolysans.
\end{abstract}

Received 22 December 2005

Revised 3 March 2006

Accepted 9 March 2006

\section{INTRODUCTION}

Bacterial IgA1 proteases are specific post-proline endopeptidases that cleave human IgA1, including its secretory form, S-IgA1, at the hinge region (reviewed by Plaut, 1983; Kilian et al., 1996). IgA1 is the predominant immunoglobulin subclass in mucosal secretions of the upper respiratory tract and is abundant in serum. Upon cleavage, the resulting Fab fragments retain antigen-binding capacity, whereas Fc-mediated effector functions are abolished. In this way, these bacteria are able to evade the protective functions of this principal mediator of adaptive immunity at mucosal surfaces and may, concomitantly, take advantage of released specific Fab fragments in facilitating adherence and immune disguise (Kilian \& Reinholdt, 1987; Kilian et al., 1996; Weiser et al., 2003). The biological significance of these enzymes is indirectly inferred by the facts that nature has developed IgA1-cleaving activity among bacteria colonizing humans

Abbreviation: RBS, ribosome-binding site.

The GenBank/EMBL/DDBJ accession numbers of the iga gene sequences from G. haemolysans strain CCUG 4815 and S. mitis strains SK564 and SK609 determined in this work are AY395568, DQ004563 and DQ004562, respectively. along at least five independent evolutionary lineages, and that all three principal bacteria causing meningitis produce an IgA1 protease (Kilian et al., 1996; Kosowska et al., 2002). Metallo-type IgA1 proteases are produced by all members of the species Streptococcus pneumoniae and by some members of the related commensal species Streptococcus sanguinis, Streptococcus oralis, Streptococcus mitis and Streptococcus infantis.

The specificity for human and great ape IgA1 implies that lack of a relevant animal model has precluded definitive proof of the role of the IgA1 proteases in pathogenesis. However, in a mouse model that did not include human IgA1, the pneumococcal IgA1 protease was identified as a virulence factor in colonization as well as in sepsis (Polissi et al., 1998; Chiavolini et al., 2003). This observation suggests that the IgA1 proteases may have features unrelated to cleavage of human IgA1 that contribute to the pathogenesis of infections caused by IgA1-protease-producing bacteria.

Sequence comparisons clearly show that the $S$. sanguinis, $S$. pneumoniae and $S$. oralis IgA1 proteases are closely related (Gilbert et al., 1991; Poulsen et al., 1996, 1998; Wani et al., 1996; reviewed by Kilian \& Reinholdt, 2005). However, 
among $S$. mitis strains, the IgA1 protease gene iga, in addition to being variably present, shows surprisingly varying degrees of homology to the other streptococcal iga genes, as revealed by Southern blot analysis (Poulsen et al., 1998). No sequence data are available to explain this polymorphism.

Gemella haemolysans is a Gram-positive, facultatively anaerobic coccus frequently found as part of the normal flora of the upper respiratory tract and the oral cavity of humans (Facklam \& Elliott, 1995), although it has been implicated in the aetiology of periodontitis (Paster et al., 2001) and is an occasional cause of subacute endocarditis and meningitis (Facklam \& Elliott, 1995). G. haemolysans has previously been shown to produce human IgA1-cleaving activity, a feature that is unique to this species within the genus Gemella (Kilian et al., 1983; Lomholt \& Kilian, 2000). The activity of the G. haemolysans IgA1 protease is similar to that of Streptococcus species, in that it cleaves at the same Pro-Thr bond in the hinge region of human IgA1 and is inhibited by EDTA, indicating that it is a metalloproteinase (Lomholt \& Kilian, 2000). Otherwise, the G. haemolysans IgAl protease has not been examined and its phylogenetic relationships to other IgA1 proteases are not known. G. haemolysans is not closely related to other bacterial species known to produce IgA1 protease activity, and the evolutionary origin of the IgA1 protease gene in this species is unknown.

To obtain further insight into the phylogeny and function of IgA1 proteases, we sequenced and characterized the iga gene encoding the IgA1 protease in G. haemolysans and in two strains of $S$. mitis.

\section{METHODS}

Bacterial strains and growth conditions. G. haemolysans strains CCUG 411, CCUG 4815, CCUG 28134, CCUG 33985 and CCUG $37985^{\mathrm{T}}$ were obtained from the Culture Collection of the University of Göteborg, Sweden, and SK940 was a dental plaque isolate from our own collection. Nine strains of $S$. mitis, four strains of $S$. oralis and four strains of $S$. infantis, including the type strains for each of the three species (NCTC 12261, NCTC 11427 and GTC 849, respectively), were from our own collection. S. pneumoniae TIGR4 was obtained from The Institute for Genomic Research, Rockville, MD. All strains except for S. mitis SK142 (NCTC $12261^{\mathrm{T}}$ ) and S. infantis SK959 (GTC $849^{\mathrm{T}}$ ) produced human IgA1-cleaving activity. Species affiliation of the streptococci was confirmed by partial sequencing of the $16 \mathrm{~S}$ rRNA genes and by phylogenetic analysis based on concatenated sequences of four housekeeping genes ( $g d h, d d l, r e c A$ and $\operatorname{sodA}$ ), as described by Hoshino et al. (2005). We have previously described the iga gene sequence of S. mitis strain SK141 (GenBank accession number Y10285) (Poulsen et al., 1998). However, recent re-evaluation of the taxonomy of the mitis group of Streptococcus reveals that this strain should be assigned to $S$. oralis (unpublished results). Therefore, we also sequenced the iga gene from two strains that were unambiguously assigned to $S$. mitis.

All strains were grown on blood agar plates and in Todd-Hewitt broth (Difco) at $37^{\circ} \mathrm{C}$ in an atmosphere supplemented with $5 \% \mathrm{CO}_{2}$. Escherichia coli strain TOP10F' (Invitrogen) was grown in $2 \times$ yeast tryptone (YT) medium (Sambrook et al., 1989) supplemented with $25 \mathrm{mg}$ tetracycline $\mathrm{l}^{-1}$ and $50 \mathrm{mg}$ ampicillin $\mathrm{l}^{-1}$ when appropriate.
IgA1 protease assay. For detection of human IgA1-cleaving activity, the sample and PBS, $\mathrm{pH} 7 \cdot 4$, up to $50 \mu \mathrm{l}$, were mixed with $2 \mu \mathrm{l}$ human myeloma IgA1 $\left(8 \mathrm{mg} \operatorname{IgA1~ml}{ }^{-1}\right)$ and incubated overnight at $37^{\circ} \mathrm{C}$, and characteristic cleavage fragments were detected by reducing SDS-PAGE of $10 \mu \mathrm{l}$ reaction mixture followed by staining with Coomassie Brilliant Blue. To differentiate between cell-associated and secreted IgAl protease activity, an overnight culture was centrifuged to separate the fractions and the bacteria were resuspended in an equal volume of PBS, $\mathrm{pH} 7 \cdot 4$. The protein synthesis was stopped by adding $20 \mathrm{mg}$ chloramphenicol $\mathrm{l}^{-1}$ prior to adding substrate IgA1 and incubation, as described above. Testing serial twofold dilutions of the fractions, combined with visual inspection of the amount of human IgA1 cleaved in the SDS-PAGE analysis, were used to quantify the IgAl protease activity. For the activity blot experiment, proteins in the supernatant were concentrated 20 times using Centriprep concentrators (Amicon) and separated by $7 \%$ SDS-PAGE under reducing conditions. IgAl protease activity in the gel upon electrophoresis was detected as described previously (Poulsen et al., 1996). In this assay, human IgAl-cleaving activity in the gel was reflected as loss of staining on the membrane.

PCR and DNA sequencing. For PCR amplification, we used $\sim 1 \mathrm{ng}$ genomic DNA as template and Ready-To-Go PCR beads (Amersham Biosciences). The temperature profile for the PCR was an initial denaturation at $94^{\circ} \mathrm{C}$ for $5 \mathrm{~min}$, followed by 30 cycles at $94{ }^{\circ} \mathrm{C}$ for $1 \mathrm{~min}, 60^{\circ} \mathrm{C}$ for $2 \mathrm{~min}, 72^{\circ} \mathrm{C}$ for $2 \mathrm{~min}$, and a final extension at $72{ }^{\circ} \mathrm{C}$ for $8 \mathrm{~min}$. When used for DNA sequencing, the resulting amplicons were purified using Wizard Minicolumns (Promega). For sequencing, we used the same primers as for the PCR, as well as primers designed from the previously determined sequence. Sequencing was achieved using the Thermo Sequenase dye terminator cycle sequencing kit (Amersham Bioscience) and the resulting products were analysed with an Applied Biosystems PRISM 377 automated sequencer (PerkinElmer/Applied Biosystems). Based on an alignment of 13 published iga gene sequences from Streptococcus species (Gilbert et al., 1991; Poulsen et al., 1996, 1998; Wani et al., 1996), we designed two degenerate primers, iga-for $5^{\prime}$-GGTAAATCWGGYTWTAACAA- $3^{\prime}$ and iga-rev $5^{\prime}$-ATGSGTCATYTCATGRGTAT-3', located within conserved regions (corresponding to positions 3949-3968 and 4707-4688, respectively, in the ORF of the S. pneumoniae strain PK81 iga gene, accession number X94909). Inverse PCR was performed as described by Ochman et al. (1988) and Kosowska et al. (2002). Briefly, restriction enzymes selected on the basis of obtained sequences were used to digest the genomic DNA prior to circularization by self-ligation, and outward-pointing oligonucleotide primers, designed from previous sequences, were used for PCR performed as described above. Direct sequencing was performed on both strands of overlapping PCR amplicons (details of the sequencing strategy are available upon request). The presence and sequences of imperfect tandem repeats were confirmed by the size of PCR products using several pairs of primers flanking the repeat region combined with sequencing using repeat-specific primers.

Heterologous expression in $\boldsymbol{E}$. coli. The oligonucleotides 5'-ATGGAAGATTTGCAATTATCAGTC- $3^{\prime}$ and $5^{\prime}$-TTTCCCACCAAATATAGATGTTCT- $3^{\prime}$ were used in PCR of genomic DNA from G. haemolysans strain CCUG 4815 to amplify a 5541 bp fragment of the ORF encoding the presumed secreted IgAl protease (see Results and Discussion). Similarly, oligonucleotide $5^{\prime}$-GAAAATAATCTCTTACTGAATTACAATAC- $3^{\prime}$ combined with $5^{\prime}$-TTTTTTATTCTCAAAAATTGAACTTCTAA- ${ }^{\prime}$, and $5^{\prime}$-GGAAAATAATCTTTTACTGAATTATAATACG-3' combined with $5^{\prime}$-TTTTTTATTCTCAAAAATTGAACTTCTAA-3' ${ }^{\prime}$, were used to amplify 5631 and 5754 bp fragments of the iga genes from S. mitis strains SK609 and SK564, respectively. We used the Expand High Fidelity PCR System (Roche Molecular Biochemicals) for the amplification as described by the 
manufacturer. The PCR products were separated by $1 \%$ agarose gel electrophoresis, visualized by staining with ethidium bromide, and DNA in the band was purified using QIAEXII (Qiagen). Adenosine overhangs were introduced by incubating with Hot Master Mix (Eppendorf) for $8 \mathrm{~min}$ at $72{ }^{\circ} \mathrm{C}$. The PCR fragments were cloned using the pBAD-TOPO TA cloning kit and E. coli TOP10F' as host (Invitrogen). The expression vector pBAD-TOPO is intended for cloning PCR products and it adds 14 aa to the $\mathrm{N}$ terminus of the recombinant protein and 28 residues, including a V5 epitope and a polyhistidine region, to the $\mathrm{C}$ terminus, and expression from the $\mathrm{P}_{\mathrm{BAD}}$ promoter is controlled by presence of arabinose. A single clone from each of the three transformations with the insert in the right orientation was selected. Expression of the recombinant protein was induced in a fresh broth culture by adding arabinose to a concentration of $0.002 \%$, as described in the manufacturer's manual. After incubation for $4 \mathrm{~h}$, the bacteria were disrupted by repeated freeze/ thawing.

Sequence analyses. For sequence comparisons and alignments, we used the program PILEUP included in the GCG package (Wisconsin Package Version 10.3; Accelrys).

For searching public sequence databases we used BLAST (available at http://www.ncbi.nlm.nih.gov/blast/). For predicting iga genes, we used the program EasyGene trained on Staphylococcus aureus as host organism (available at http://www.cbs.dtu.dk/services/EasyGene/) (Larsen \& Krogh, 2003). For predicting signal peptides, we used the program SignalP (available at http://www.cbs.dtu.dk/services/SignalP) (Nielsen et al., 1997). Transmembrane segments were predicted by the program DAS (available at http://www.sbc.su.se) (Cserzö et al., 1997). Protein domains were predicted by the program Pfam (available at http://pfam.wustl.edu/) (Bateman et al., 2004). For construction of dendrograms based on the neighbour-joining method, we used MEGA3 (available at http://www.megasoftware.net/) (Kumar et al., 2004).

Southern blot analysis. Whole-cell DNA was purified as described by Hohwy \& Kilian (1995). Approximately $10 \mu \mathrm{g}$ DNA was digested with EcoRI and treated with RNase. DNA fragments were separated by $1 \%$ agarose gel electrophoresis and transferred to and fixed onto a nylon membrane, as described by Sambrook et al. (1989). For detection of iga gene sequences on the blot, we used the Gene Images Alkphos Direct Labelling and Detection System (Amersham Bioscience). The six probes used in the Southern blot experiments were equivalent fragments of the 3' part of the iga genes from G. haemolysans CCUG 4815, S. mitis SK564 and S. pneumoniae TIGR4. The fragments were CCUG 4815-1 and CCUG 4815-2 (positions 4165-4658 and 5577-5906, respectively, in the ORF), SK564-1 and SK564-2 (positions 4354-4844 and 5778-6119, respectively, in the ORF), and TIGR4-1 and TIGR4-2 (positions 4159-4649 and 5577-5915, respectively, in the ORF).

\section{RESULTS AND DISCUSSION}

\section{The IgA1 proteases of $S$. mitis and G. haemolysans}

Two strains of S. mitis, SK564 and SK609, and G. haemolysans strain CCUG 4815, all with IgA1 protease activity, were included in this part of the study. Pronounced differences in the amount of cell-associated versus secreted IgA1 protease activity have been demonstrated among different species of bacteria (Reinholdt \& Kilian, 1997). In the three strains included in the present study, the greater part $(>80 \%)$ of the IgAl protease activity was present in the supernatant of an overnight culture (results not shown). In an activity blot experiment performed upon separation of secreted proteins by reducing SDS-PAGE, the IgA1 protease was present in multiple molecular forms (Fig. 1). In all three strains, the largest protein in the supernatant with human IgA1-cleaving activity had an apparent molecular mass of $\sim 220 \mathrm{kDa}$ (Fig. 1). This is very similar to what has previously been observed for other streptococcal IgA1 proteases (Poulsen et al., 1996, 1998). For the S. pneumoniae IgA1 protease, we showed that the smaller molecular forms emerged during concentration and prolonged storage, while fresh cultures only showed the largest forms (unpublished results). Autoproteolysis was presumably the cause of this breakdown of the protein into smaller sizes that retained IgA1 protease activity.

\section{Sequencing S. mitis and G. haemolysans iga genes}

PCR amplification using the primers iga-for and iga-rev on genomic DNA from S. mitis strains SK564 and SK609 and G. haemolysans strain CCUG 4815 as template resulted in a fragment of $\sim 0.7 \mathrm{~kb}$. Sequencing of the resulting amplicons revealed an ORF in all three bacteria with similarity to the known streptococcal iga genes, suggesting that the amplified fragments represented the homologous iga genes. The sequences of the remaining parts of the presumed iga genes and flanking regions were obtained by 'walking' in both directions using inverse PCR. For S. mitis strains SK564 and SK609 and for G. haemolysans strain CCUG 4815, continuous sequences of 7054, 7553 and $7453 \mathrm{nt}$, respectively, were obtained.

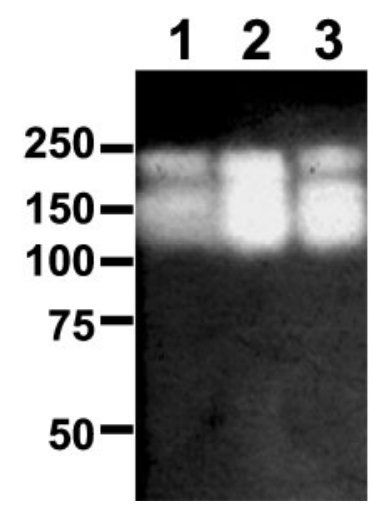

Fig. 1. $\lg A 1$ protease profiles. Proteins in the supernatant concentrated 20 times were separated according to size by reducing SDS-PAGE. Proteins in the gel were renatured and allowed to react with human $\lg A 1$ bound to a filter via the $\mathrm{FC}$ region. IgA1-cleaving activity diffusing out of the gel was visualized on the filter as lack of reaction with anti-light chain antibodies. Lane 1, G. haemolysans strain CCUG 4815; lane 2, S. mitis strain SK609; lane 3, S. mitis strain SK564. Molecular mass markers in $\mathrm{kDa}$ are shown to the left. 
For S. mitis SK564, the sequence revealed an ORF of $6216 \mathrm{nt}$, starting with ATG, and with the potential of encoding a protein of 2072 aa. The S. mitis SK609 sequence contained an ORF of $6093 \mathrm{nt}$, also starting with ATG and encoding a protein of 2031 aa. The ORFs of both $S$. mitis strains showed significant similarity to known streptococcal IgA1 proteases. In both strains, the ORF was preceded by a typical ribosomebinding site (RBS), AAGGAGGA, 3 nt upstream of the proposed start codon. Just upstream of this, -10 and -35 consensus promoter sequences were present. A possible weak transcription terminator with a stem of $6 \mathrm{nt}$ and a loop of $3 \mathrm{nt}$ was present in both sequences, $65 \mathrm{nt}$ downstream of the stop codon. In both sequences the ORF was predicted to represent a real gene by the program EasyGene. In the same orientation and $209 \mathrm{nt}$ upstream of the ORF, both strains harboured an ORF with homology to the C-terminal part of a gene encoding a conserved hypothetical protein, preceding the iga gene in the published genome sequences of $S$. pneumoniae strains TIGR4 and R6. In S. mitis strain NCTC $12261^{\mathrm{T}}$, which lacks IgA1 protease activity, the order of genes was as in the pneumococci, except that the iga gene was missing (Fig. 2). In both S. mitis SK564 and SK609, the sequence downstream of the ORF was homologous to that downstream of the iga gene in S. pneumoniae strain PK81 (GenBank accession number X94909), including an ORF with homology to the $5^{\prime}$ end of the paralogous zinc metalloproteinase $\mathrm{B}$ gene $z m p B$ and iga. A similar gene organization, with the iga gene followed by an IgA1-protease-like gene termed $z m p D$, has been found in S. pneumoniae strain G54 (Polissi et al., 1998), strain PK81 (Poulsen et al., 1996), and approximately half of clinical isolates of $S$. pneumoniae (Camilli et al., 2006), whereas this apparent gene duplication is absent in strains TIGR4 and R6. In summary, the gene order in the region around iga was conserved between S. mitis and certain S. pneumoniae strains (Fig. 2).

For G. haemolysans strain CCUG 4815, the combined sequence information revealed an ORF of $6051 \mathrm{nt}$, starting at the first ATG codon and with significant similarity to the streptococcal iga genes. The sequences TTGCAT and TACAAT, separated by $17 \mathrm{nt}$ and located $18 \mathrm{nt}$ upstream of the ORF, have similarity to the -35 and -10 elements of the consensus promoter sequence (Hawley \& McClure, 1983). However, the first ATG in the ORF was not preceded by a possible RBS. We propose that the TTG sequence located 51 nt downstream of this ATG codon serves as a start codon in the G. haemolysans iga gene, because of the high degree of similarity with known streptococcal IgA1 proteases at the $\mathrm{N}$ terminus, combined with the presence of the putative RBS GAAGGA, 7 nt upstream of the TTG triplet. TTG is often used as a start codon in bacteria (Kozak, 1999). In addition, the program EasyGene predicted this ORF to be a real gene. The ORF has the potential of encoding a protein of 2000 aa. Twenty-six nucleotides downstream, the ORF is followed by a possible transcription terminator, consisting of an inverted repeat structure with a stem of $13 \mathrm{nt}$ and a loop of 13 nt. Putative genes flanking the large ORF, and in the same orientation, were identified at the ends of the sequence obtained. At the upstream end, separated from the proposed iga gene by $263 \mathrm{nt}, 621 \mathrm{nt}$ of an ORF encoding a protein with $70 \%$ identity to the C-terminal part of an ABC transporter from Streptococcus agalactiae were present. Five nucleotides downstream of this ORF, an inverted repeat structure, which may constitute a transcription terminator, was identified. At the downstream end, separated from the iga gene by $166 \mathrm{nt}$, there was an ORF of $397 \mathrm{nt}$ encoding a

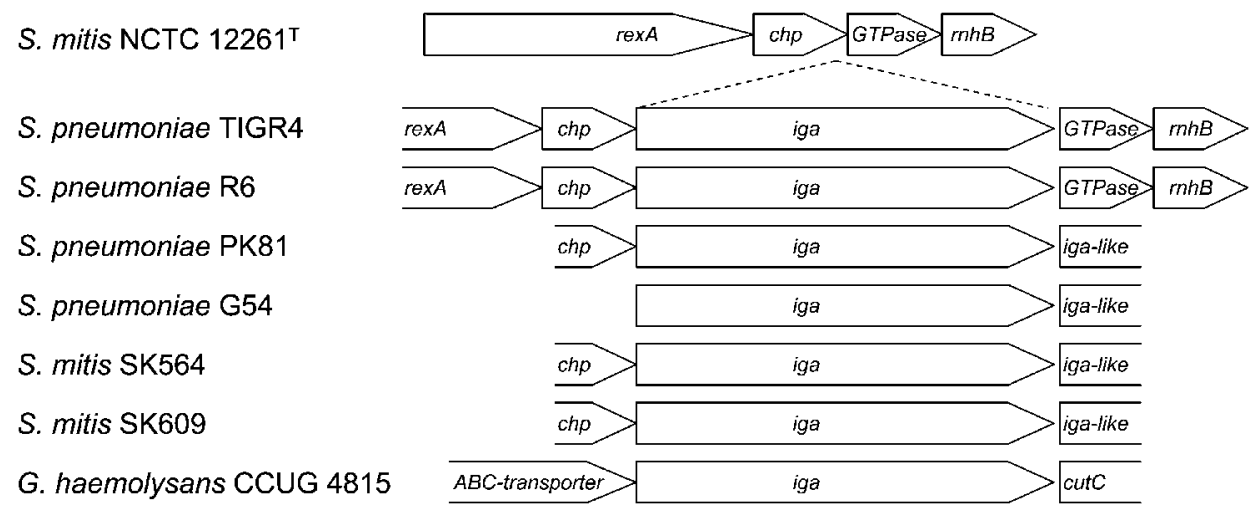

Fig. 2. Order of genes in the iga gene region. S. mitis NCTC $12261^{\top}$ did not encode $\lg A 1$ protease activity, in contrast to all the other strains included. ORFs flanking individual iga genes were deduced from complete genome sequences ( $S$. pneumoniae strains TIGR4 and R6), unfinished genome sequences of S. mitis NCTC $12261^{\top}$ (available at http:// www.tigr.org), Poulsen et al. (1996) (S. pneumoniae PK81), Polissi et al. (1998) (S. pneumoniae G54) and sequences obtained in this study. Flanking genes were: rexA, first chain of major exonuclease gene; chp, the same conserved hypothetical protein gene; GTPase, GTP binding protein gene; $r n h B$, ribonuclease HIl gene; iga-like, ORF with homology to $\lg \mathrm{A} 1$ protease and zinc metalloproteinase $\mathrm{B} ; A B C$-transporter, ATP binding protein gene; cutC, copper homeostasis protein gene. 
protein with up to $42 \%$ amino acid identity to the $\mathrm{N}$ terminus of a copper homeostasis protein, CutC, found in a variety of prokaryotes and eukaryotes. This ORF was preceded by a typical ribosome-binding sequence GGAGAA, $6 \mathrm{nt}$ upstream of the ATG start codon. Thus, there was no synteny of the area of the genome containing the iga gene in G. haemolysans compared to S. mitis and S. pneumoniae (Fig. 2).

\section{Heterologous expression of the $\mathbf{S}$. mitis and G. haemolysans iga genes in E. coli}

In S. pneumoniae, the iga gene is part of a gene family that includes at least two other paralogous members, $z m p B$ and $z m p C$. Among these zinc metalloproteinases, only the IgA1 protease cleaves human IgA1 (Oggioni et al., 2003). A higher degree of similarity to iga than $z m p B$ and $z m p C$ suggested that the three sequenced ORFs represented the IgA1 protease in S. mitis SK564, S. mitis SK609 and G. haemolysans CCUG 4815 (see below). In order to verify this, we performed heterologous expression in E. coli. For each of the three bacteria, the part of the ORF encoding the presumed secreted version of the IgA1 protease (see below) was amplified by PCR. The PCR products corresponded to the sequences encoding amino acid positions 154-2072, 154-2031 and 155-2000 in S. mitis SK564, S. mitis SK609 and G. haemolysans CCUG 4815, respectively. The amplicons were cloned into the expression vector pBAD using E. coli $\mathrm{TOP} 10 \mathrm{~F}^{\prime}$ as host. The resulting recombinant plasmids with the inserts in the right orientation were termed pBAD-SK564-iga, pBAD-SK609-iga and pBAD-gemella-iga. Expression of the recombinant proteins was induced by adding arabinose. After incubation the bacteria were disrupted by repeated freezing and thawing. Each of the three resulting lysates possessed human IgA1-cleaving activity, demonstrating that the three sequenced ORFs encode the IgA1 protease (Fig. 3).

\section{Structural features of the S. mitis and G. haemolysans IgA1 proteases}

The deduced primary structure of the S. mitis and G. haemolysans IgA1 proteases shared a number of sequence motifs with known orthologous enzymes of streptococci (Fig. 4). The amino acid sequence HEMTH followed by an $\mathrm{E}$ residue 20 aa downstream is typical of bacterial metalloproteinases of the gluzincin type (Vallee \& Auld, 1990; Hooper, 1994), and the IgA1 proteases belong to the clan MA(E) family M26 of proteases listed in the MEROPS database (available at http://merops.sanger.ac.uk).

The signal peptides of the S. mitis SK564 and SK609 IgA1 proteases each comprised 42 aa, as predicted by the program SignalP. The size of the signal peptide predicted for the IgA1 protease of G. haemolysans CCUG 4815 was 44 aa. The presence of a cysteine at position 22 in the G. haemolysans IgA1 protease might suggest that it is a lipoprotein, but the sequence upstream of this residue did not match the consensus pattern for signal peptides of lipoproteins in
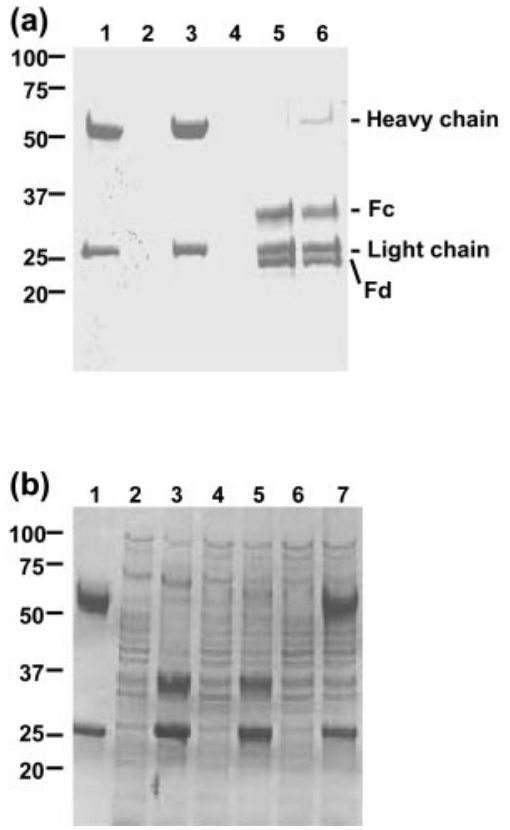

Fig. 3. The recombinant $\lg A 1$ proteases from $G$. haemolysans CCUG4815 and S. mitis SK564 and SK609 have human $\lg A 1$-cleaving activity. Expression of recombinant proteins in E. coli TOP10F' harbouring plasmids was induced by adding arabinose, incubating, and lysing, as described in the text. Samples were incubated overnight at $37^{\circ} \mathrm{C}$, analysed by reducing SDS-PAGE, and proteins in the gel were visualized by staining with Coomassie Blue. (a) Lane 1, $5 \mu \mathrm{g}$ human IgA1; lane 2, $1 \mu \mathrm{l}$ lysate of $E$. coli harbouring the $\mathrm{pBAD}$ vector; lane $3,1 \mu$ lysate of $E$. coli harbouring the pBAD vector incubated with human IgA1; lane $4,1 \mu$ lysate of $E$. coli harbouring plasmid pBAD-gemella-iga; lane $5,1 \mu$ lysate of $E$. coli harbouring plasmid pBAD-gemella-iga incubated with human $\lg A 1$; lane 6 , supernatant of $G$. haemolysans strain CCUG 4815 incubated with human $\lg \mathrm{A} 1$. Molecular mass markers in $\mathrm{kDa}$ are shown to the left. $\mathrm{Fc}$ and $\mathrm{Fd}$ indicate the $\mathrm{C}$ - and $\mathrm{N}$-terminal fragments upon cleavage of the heavy chain, respectively. (b) Lane 1, $5 \mu \mathrm{g}$ human $\operatorname{lgA} 1$; lane 2, $10 \mu \mathrm{l}$ lysate of $E$. coli harbouring plasmid pBAD-SK564-iga; lane 3, as in lane 2, incubated with $\lg A 1$; lane $4,10 \mu$ lysate of $E$. coli harbouring plasmid pBADSK609-iga; lane 5, as in lane 4, incubated with $\operatorname{lgA} 1$; lane 6 , $10 \mu$ lysate of $E$. coli harbouring plasmid PBAD vector; lane 7, as in lane 6 , incubated with $\lg A 1$. Different preparations of human $\lg A 1$ with a distinct glycosylation were used in (a) and (b); therefore, the fragments migrated slightly differently in the gel [in (b) the Fd fragment migrated together with the light chain].

Gram-positive bacteria (Sutcliffe \& Harrington, 2002). Otherwise, all three IgA1 proteases were devoid of cysteine residues. The streptococcal and $G$. haemolysans signal peptides included a sequence similar to the motif YSIRK$\mathrm{G} / \mathrm{S}$, which is associated with proteins carrying a Grampositive anchor domain at the $\mathrm{C}$ terminus (Tettelin et al., 2001; Bae \& Schneewind, 2003). 


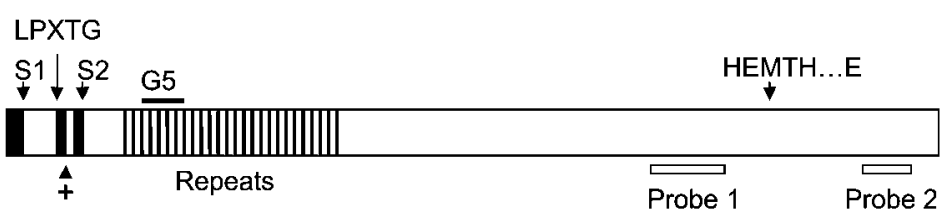

Fig. 4. Schematic structure of the streptococcal and $G$. haemolysans $\lg A 1$ proteases. Black boxes indicate hydrophobic regions proposed to serve as transmembrane domains in the signal peptide and in combination with the cell-wall-anchor motif LPXTG. S1 and S2 indicate sites presumably cleaved by the signal peptidase. The G5 domain conserved in the streptococcal $\lg \mathrm{A} 1$ proteases and location of the amino acids encoded by the two probes used in the Southern blot analysis are indicated.
In the two S. mitis and the G. haemolysans IgA1 proteases, as well as in all other known streptococcal IgA1 proteases, a cell-wall-anchor motif LPNTG (LPYTG in S. mitis strain SK609) (Navarre \& Schneewind, 1999; Pallen et al., 2001) is present 53 aa downstream of the proposed signal peptidase site, and followed by two hydrophobic domains with the potential of spanning the cell membrane (Fig. 4). The region between the two putative transmembrane segments is rich in lysines and, according to the positive-inside rule, this region is predicted to face the cytoplasm (von Heijne, 1989). We have previously hypothesized that the streptococcal protease is cleaved at the LPNTG sortase processing site at its $\mathrm{N}$ terminus, traverses the cytoplasmic membrane twice, and is released by proteolysis (Poulsen et al., 1998). Nterminal sequence analysis of the $S$. pneumoniae strain PK81 IgA1 protease showed that it is cleaved at the $\mathrm{Ala}^{153}-\mathrm{Leu}^{154}$ peptide bond, just after the last hydrophobic region, presumably by the signal peptidase enzyme (unpublished results). Cleavage of the S. mitis SK564, S. mitis SK609 and G. haemolysans CCUG 4815 IgA1 proteases after the corresponding alanine would result in secreted proteases with molecular masses of $213415 \mathrm{Da}$ with a pI of $5 \cdot 01$, 209722 Da with a pI of $5 \cdot 13$, and 205586 Da with a pI of $5 \cdot 1$, respectively. The proposed processing is in agreement with results from the activity blot experiment, which showed that the largest species of secreted proteins with human IgAl-cleaving activity in each of the three strains had an apparent molecular mass of $\sim 220 \mathrm{kDa}$.

Following the putative cleavage site, the three IgA1 proteases contained 18-23 imperfect repeats, consisting of repeat sequences 16-20 aa in length. Repeat structures are commonly found in surface-exposed and secreted proteins in Gram-positive bacteria, and variation in the number and sequence of repeats may contribute to antigenic variation and thereby to immune evasion (Kehoe, 1994). The repeat structure in the $S$. sanguinis IgA1 protease has been found to be immunodominant and non-essential for enzyme activity (Gilbert et al., 1993).

In contrast to that of G. haemolysans, all the streptococcal IgA1 proteases harboured a sequence with similarity to the G5 domain, which has been suggested to be a lectin that binds $N$-acetylglucosamine (Bateman et al., 2005). The sequences contained four of the five conserved glycine residues in the consensus G5 domain (Bateman et al., 2005). This domain was located within the otherwise highly variable repeat structure, suggesting that it has an essential function. In S. pneumoniae, the greater part of the IgA1 protease is non-covalently bound to the cell wall (unpublished results). Further experiments are needed to identify the potential ligand of this domain.

\section{Southern blot analysis}

We previously found that the iga gene sequences of $S$. sanguinis and $S$. oralis are highly conserved within each of the two species, whereas Southern blot analysis has revealed extensive gene polymorphism among $S$. pneumoniae and S. mitis strains (Poulsen et al., 1996, 1998). Hakenbeck et al. (2001) have also shown variation in the iga gene among $S$. pneumoniae strains. Here, we used iga gene probes from $G$. haemolysans CCUG 4815, S. mitis SK564 and S. pneumoniae TIGR4 in Southern blot analyses to assay for the degree of similarity in iga genes within and between the species $G$. haemolysans, S. mitis, S. oralis and S. infantis. The two probes from each of the three strains represented equivalent areas flanking the active site (encoding amino acid positions 1388-1552 and 1859-1968 in the G. haemolysans CCUG 4815 IgA1 protease; Fig. 4). An example of the Southern blot analysis is shown in Fig. 5 and the results are compiled in Table 1. The iga genes, including the areas covered by the probes, are supposed to be homologous. Lack of hybridization for some of the probe/genomic DNA combinations under the stringency conditions used suggests lack of significant similarity in the DNA sequences. For example, the 490 bp SK564-1 probe sequence showed only $74 \%$ similarity to the G. haemolysans CCUG 4815 iga gene in a $132 \mathrm{bp}$ segment without longer stretches with identity, and the 611 bp SK564-2 probe had no significant similarity to the gemella iga gene. A potential complication in interpreting the hybridization results is the presence in S. pneumoniae and S. mitis genomes, and presumably also in some of the other species, of paralogues of the iga gene with varying degrees of homology (Kilian \& Reinholdt, 2005). However, the specificity of the hybridization is supported by the fact that $S$. mitis strain SK142 (NCTC 12261), which lacks the iga gene but has two paralogous 
(a)

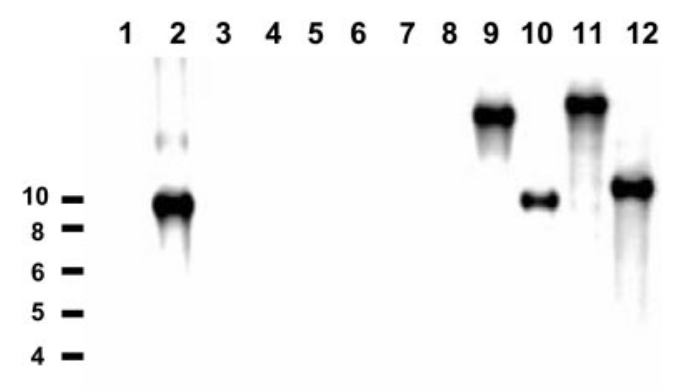

(b)

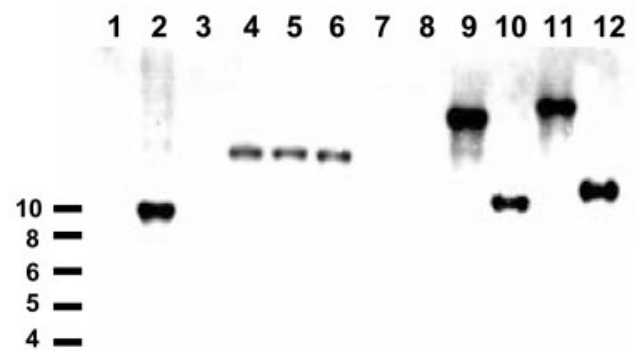

Fig. 5. Example of Southern blot analysis. Whole-cell DNA was restricted with EcoRl, electrophoresed in an agarose gel, transferred to a nylon membrane, and hybridized with the SK564-1 (a) and SK564-2 (b) probes. Lane 1, SK142; lane 2, SK564; lane 3, SK599; lane 4, SK602; lane 5, SK608; lane 6, SK609; lane 7, SK612; lane 8, SK675 all of S. mitis; lane 9, SK2; lane 10, SK23; lane 11, SK141; lane 12, SK286 all of $S$. oralis. Molecular mass markers in $\mathrm{kb}$ are shown to the left. In (a), lanes 2, 9, 10, 11 and 12 were visually scored as strong hybridization and lanes $1,3,4,5,6,7$ and 8 as no hybridization. In (b), lanes 2, 9, 10, 11 and 12 were scored as strong; lanes 4, 5 and 6 as weak; and lanes 1, 3, 7 and 8 as no hybridization.

genes (unfinished genome sequence available at http:// www.tigr.org), showed no hybridization.

The intensity of hybridization with the different probes was very similar for the six strains of $G$. haemolysans, indicating that the iga gene is highly conserved within this species. The same applied to the four strains of $S$. oralis. The eight strains of $S$. mitis with IgA1 protease activity showed varying degrees of hybridization, with the iga gene probes indicating significant sequence diversification of the iga gene within this species. This is supported by the high degree of dissimilarity between the two $S$. mitis iga genes sequenced in this study (see below). Notably, strong hybridization with the G. haemolysans iga gene was common among the S. mitis strains. The sequence analysis and the Southern blotting show that the G. haemolysans iga gene is very similar to the iga gene found in certain $S$. mitis strains. We also included four strains of the species S. infantis (Kawamura et al., 1998), three of which produced IgA1 protease activity. None of the $S$. infantis strains showed hybridization to any of the probes, indicating that the IgAl protease-positive strains of this species have a more divergent iga gene sequence. Though highly unlikely, it is a theoretical possibility that the S. infantis iga gene may not be evolutionarily related and therefore not homologous to iga genes of the other streptococci and G. haemolysans.

\section{Phylogenetic considerations}

The genera Streptococcus and Gemella both belong to the Gram-positive bacteria with a low $\mathrm{G}+\mathrm{C}$ content. Phylogeny based on $16 \mathrm{~S}$ rRNA sequences indicates that Gemella is more closely related to the Bacillus-Staphylococcus complex than to streptococci and lactobacilli. IgA1 protease with significant sequence similarity to that of G. haemolysans described here has been exclusively found among Streptococcus species of the mitis group (Kilian et al., 1996; Kilian \& Reinholdt, 2005). A likely explanation of the distribution of this finding is horizontal gene transfer, rather than vertical inheritance from a common ancestor combined with subsequent loss of the gene in some species. Deviation in $\mathrm{G}+\mathrm{C}$ content of a gene compared to the whole genome is often a valuable marker for identifying genes acquired recently by horizontal transfer. The G $+\mathrm{C}$ content of the G. haemolysans iga gene was $34 \%$ which is very similar to the $33-35 \%$ reported for the genome (Reyn, 1986). The streptococci displaying IgA1 protease activity have a genome $\mathrm{G}+\mathrm{C}$ content of $38-46 \%$ and the content for their iga genes varies from 34 to $41 \%$.

Table 1. Patterns of hybridization with iga gene probes

$\mathrm{N}$, no hybridization; S, strong hybridization; W, weak hybridization.

\begin{tabular}{|c|c|c|c|c|c|c|c|c|}
\hline \multirow[t]{2}{*}{ Species } & \multirow{2}{*}{$\begin{array}{l}\text { No. of } \\
\text { strains }\end{array}$} & \multirow{2}{*}{$\begin{array}{l}\text { IgA1 protease } \\
\text { activity }\end{array}$} & \multicolumn{6}{|c|}{ Probe used for hybridization } \\
\hline & & & CCUG4815-1 & CCUG4815-2 & SK564-1 & SK564-2 & TIGR4-1 & TIGR4-2 \\
\hline S. mitis & 1 & - & $\mathrm{N}$ & $\mathrm{N}$ & $\mathrm{N}$ & $\mathrm{N}$ & $\mathrm{N}$ & $\mathrm{N}$ \\
\hline S. mitis & 8 & + & S/W & $\mathrm{W} / \mathrm{N}$ & $\mathrm{S} / \mathrm{N}$ & $\mathrm{S} / \mathrm{W} / \mathrm{N}$ & $\mathrm{W} / \mathrm{N}$ & $\mathrm{W} / \mathrm{N}$ \\
\hline S. oralis & 4 & + & $\mathrm{N}$ & W & S & S & W & W \\
\hline S. infantis & 3 & + & $\mathrm{N}$ & $\mathrm{N}$ & $\mathrm{N}$ & $\mathrm{N}$ & $\mathrm{N}$ & $\mathrm{N}$ \\
\hline
\end{tabular}




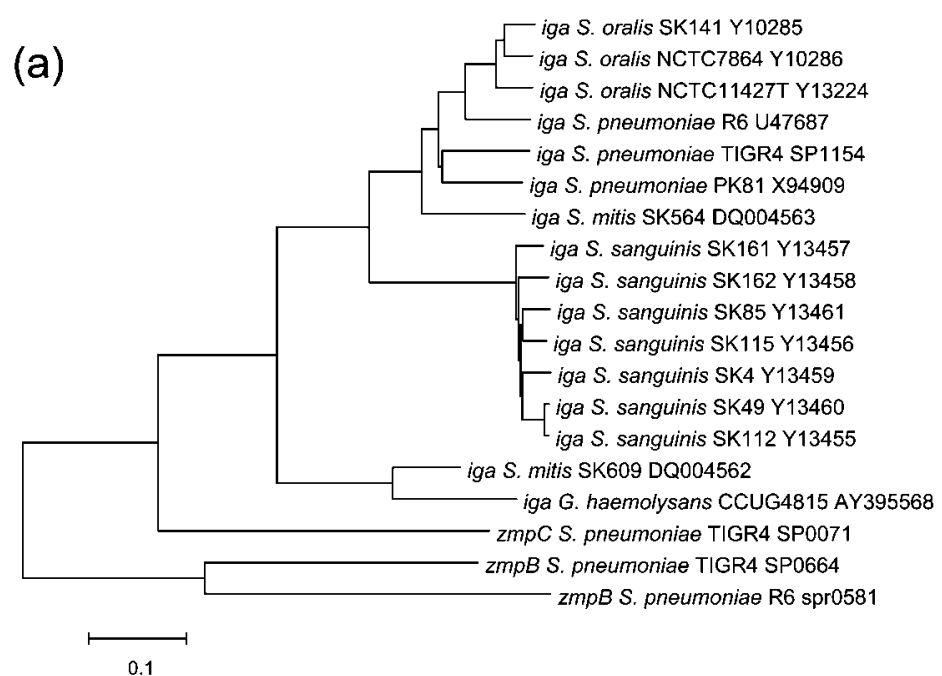

(b)

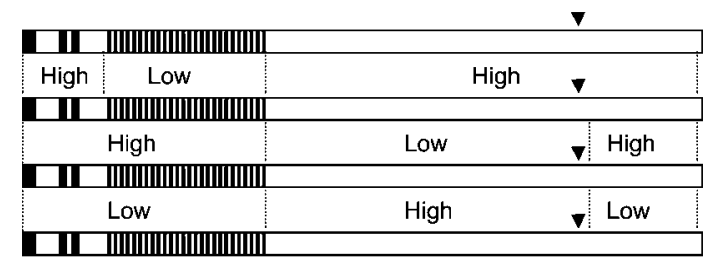

S. pneumoniae PK81

S. mitis SK564

S. mitis SK609

G. haemolysans 4815
Fig. 6. (a) Dendrogram illustrating proportional similarity in DNA sequences among homologous iga, zmpB and $z m p C$ gene sequences from streptococcal species and G. haemolysans. The analysis included sequences encoding the last two-thirds of the proteins (position 1914-5783 in the ORF of the $S$. pneumoniae PK81 iga gene), thus excluding the repeat region. Species affiliation, strain designation and accession/ gene identification number are shown for each sequence. (b) Distribution of mutual sequence homologies, indicated as high and low, along the $\lg A 1$ proteases, illustrating the mosaic structure. The active site is shown by a triangle.
However, because Gemella and Streptococcus have similar low $\mathrm{G}+\mathrm{C}$ content, it is not possible from these data to draw conclusions on the origin of the iga gene. Several other observations support the hypothesis that the iga gene is transferred from Streptococcus to G. haemolysans. The iga gene is apparently ancient within the mitis group of the genus Streptococcus. This is supported by its presence in several closely related species and by the conserved gene order in the iga gene region of S. pneumoniae and S. mitis, which is different from that in G. haemolysans (Fig. 2). An ancestral streptococcus with an iga gene is also supported by the significant sequence diversification resulting in speciesspecific clusters of iga gene sequences (Fig. 6). In the genus Gemella, only G. haemolysans displays an iga gene, and the Southern blot analysis indicates that the gene is highly conserved within this species. This argues strongly for the transfer of the iga gene from a mitis group Streptococcus to the ancestor of G. haemolysans after its separation from other Gemella species.

A subsequent, evolutionarily recent, homologous horizontal gene transfer has apparently taken place between S. mitis and G. haemolysans since the S. mitis SK609 and G. haemolysans CCUG 4815 iga genes showed $89 \%$ identity in a $2 \cdot 8 \mathrm{~kb}$ DNA sequence encoding from the end of the repeats down to the active site, whereas the areas flanking this region were more different in the two bacteria. The mosaic structure of the mutual homologies among the IgA1 proteases is shown in Fig. 6(b). This was reflected in the Southern blot hybridizations. The CCUG4815-1 probe located upstream of the active site hybridized strongly to S. mitis SK609 genomic DNA, whereas the CCUG4815-2 probe downstream of the active site only showed weak hybridization to SK609 (Fig. 4, Table 1). This pattern of hybridization with the G. haemolysans iga gene probes was detected in five of eight IgA1 protease-positive strains of S. mitis, suggesting that the SK609 iga gene version is common among members of this species.

\section{Concluding remarks}

The sequence of the G. haemolysans IgA1 protease was homologous to streptococcal IgA1 proteases that belong to a family of zinc metalloproteinases found in the mitis group of Streptococcus. The genus Gemella is only distantly related to Streptococcus and we argue that the iga gene in G. haemolysans was acquired from Streptococcus by horizontal gene transfer. Structural features conserved among members of this family of IgA1 proteases may delineate sequences essential for enzymic function, including mechanisms of secretion and substrate specificity. Heterologous expression in E. coli allows for purification and manipulation and therefore further studies of these intriguing proteases.

\section{ACKNOWLEDGEMENTS}

Financial support was obtained from the Danish Medical Research Council grant 22-01-0265 ct/mp and Aarhus University Research Foundation. L. M.M. and K.R.D. both received a Novo student 
scholarship. N.T.-O. was supported by the Sato Fund, Nihon University School of Dentistry, and the Overseas Researchers (Academic Year 2004), Nihon University.

\section{REFERENCES}

Bae, T. \& Schneewind, O. (2003). The YSIRK-G/S motif of staphylococcal protein A and its role in efficiency of signal peptide processing. J Bacteriol 185, 2910-2919.

Bateman, A., Coin, L., Durbin, R. \& 10 other authors (2004). The Pfam protein families database. Nucleic Acids Res 32, D138-D141.

Bateman, A., Holden, M. T. G. \& Yeats, C. (2005). The G5 domain: a potential $\mathrm{N}$-acetylglucosamine recognition domain involved in biofilm formation. Bioinformatics 21, 1301-1303.

Camilli, R., Pettini, E., Del Grosso, M., Pozzi, G., Pantosti, A. \& Oggioni, M. R. (2006). Zinc metalloproteinase genes in clinical isolates of Streptococcus pneumoniae: association of the full array with a clonal cluster comprising serotypes 8 and 11A. Microbiology 152, 313-321.

Chiavolini, D., Memmi, G., Maggi, T., lannelli, F., Pozzi, G. \& Oggioni, M. R. (2003). The three extra-cellular zinc metalloproteinases of Streptococcus pneumoniae have a different impact on virulence in mice. BMC Microbiol 3, 14.

Cserzö, M., Wallin, E., Simon, I., von Heijne, G. \& Eloffson, A. (1997). Prediction of transmembrane $\alpha$-helices in prokaryotic membrane proteins: the dense alignment surface method. Prot Eng 10, 673-676.

Facklam, R. \& Elliott, J. A. (1995). Identification, classification, and clinical relevance of catalase-negative, gram-positive cocci, excluding the streptococci and enterococci. Clin Microbiol Rev 8, 479-495.

Gilbert, J. V., Plaut, A. G. \& Wright, A. (1991). Analysis of the immunoglobulin A protease gene of Streptococcus sanguis. Infect Immun 59, 7-17.

Gilbert, J. V., Ramakrishna, J. P., Wright, A. \& Plaut, A. G. (1993) Streptococcal IgA protease tandem repeat influences antigenicity but not activity. J Dent Res 72, 327.

Hakenbeck, R., Balmelle, N., Weber, B., Gardes, C., Keck, W. \& de Saizieu, A. (2001). Mosaic genes and mosaic chromosomes: intraand interspecies genomic variation of Streptococcus pneumoniae. Infect Immun 69, 2477-2486.

Hawley, D. K. \& McClure, W. R. (1983). Compilation and analysis of Escherichia coli promoter DNA sequences. Nucleic Acids Res 11, 2237-2255.

Hohwy, J. \& Kilian, M. (1995). Clonal diversity of the Streptococcus mitis biovar 1 population in the human oral cavity and pharynx. Oral Microbiol Immunol 10, 19-25.

Hooper, N. M. (1994). Families of zinc metalloproteases. FEBS Lett 354, 1-6.

Hoshino, T., Fujiwara, T. \& Kilian, M. (2005). Use of phylogenetic and phenotypic analyses to identify nonhemolytic streptococci isolated from bacteremic patients. J Clin Microbiol 43, 6073-6085.

Kawamura, Y., Hou, X.-G., Todome, Y., Sultana, F., Hirose, K., Shu, S.-E., Ezaki, T. \& Ohkuni, H. (1998). Streptococcus peroris sp. nov. and Streptococcus infantis sp. nov., new members of the Streptococcus mitis group, isolated from human clinical specimens. Int J Syst Bacteriol 48, 921-927.

Kehoe, M. A. (1994). Cell-wall-associated proteins in Gram-positive bacteria. In Bacterial Cell Wall, pp. 217-261. Edited by J.-M. Ghuysen \& R. Hakenbeck. New York: Elsevier

Kilian, M. \& Reinholdt, J. (1987). A hypothetical model for the development of invasive infection due to IgA1 protease-producing bacteria. In Recent Advances in Mucosal Immunology, part B, pp. 1261-1269. Edited by J. Mestecky, J. R. McGhee, J. Bienenstock \& P. L. Ogra. New York: Plenum.

Kilian, M. \& Reinholdt, J. (2005). Immunoglobulin A1 proteases of pathogenic and commensal bacteria of the respiratory tract. In Colonization of Mucosal Surfaces, pp. 119-129. Edited by J. P. Nataro, P. S. Cohen, H. L. T. Mobley \& J. N. Weiser. Washington, DC: American Society for Microbiology.

Kilian, M., Thomsen, B., Petersen, T. E. \& Bleeg, H. S. (1983). Occurrence and nature of bacterial IgA proteases. Ann N Y Acad Sci 409, 612-624

Kilian, M., Reinholdt, J., Lomholt, H., Poulsen, K. \& Frandsen, E. V. (1996). Biological significance of IgAl proteases in bacterial colonization and pathogenesis: critical evaluation of experimental evidence. APMIS 104, 321-338.

Kosowska, K., Reinholdt, J., Rasmussen, L. K., Sabat, A., Potempa, J., Kilian, M. \& Poulsen, K. (2002). The Clostridium ramosum IgA1 proteinase represents a novel type of metalloendopeptidase. J Biol Chem 277, 11987-11994.

Kozak, M. (1999). Initiation of translation in prokaryotes and eukaryotes. Gene 234, 187-208.

Kumar, S., Tamura, K. \& Nei, M. (2004). MEGA3: integrated software for Molecular Evolutionary Genetics Analysis and sequence alignment. Brief Bioinform 5, 150-163.

Larsen, T. S. \& Krogh, A. (2003). EasyGene - a prokaryotic gene finder that ranks ORFs by statistical significance. BMC Bioinformatics $4,21$.

Lomholt, J. \& Kilian, M. (2000). Immunoglobulin A1 protease activity in Gemella haemolysans. J Clin Microbiol 38, 2760-2762.

Navarre, W. W. \& Schneewind, O. (1999). Surface proteins of grampositive bacteria and mechanisms of their targeting to the cell wall envelope. Microbiol Mol Biol Rev 63, 174-229.

Nielsen, H., Engelbrecht, J., Brunak, S. \& von Heijne, G. (1997). Identification of prokaryotic and eukaryotic signal peptides and prediction of their cleavage sites. Protein Eng 10, 1-6.

Ochman, H., Gerber, A. S. \& Hartl, D. L. (1988). Genetic applications of an inverse polymerase chain reaction. Genetics 120, 621-623.

Oggioni, M. R., Memmi, G., Maggi, T., Chiavolini, D., lannelli, F. \& Pozzi, G. (2003). Pneumococcal zinc metalloproteinase ZmpC cleaves human matrix metalloproteinase 9 and is a virulence factor in experimental pneumonia. Mol Microbiol 49, 795-805.

Pallen, M. J., Lam, A. C., Antonio, M. \& Dunbar, K. (2001). An embarrassment of sortases - a richness of substrates? Trends Microbiol 9, 97-101.

Paster, B. J., Boches, S. K., Galvin, J. L., Ericson, R. E., Lau, C. N., Levanos, V. A., Sahasrabudhe, A. \& Dewhirst, F. E. (2001). Bacterial diversity in human subgingival plaque. J Bacteriol 183, 3770-3783.

Plaut, A. G. (1983). The IgA1 proteases of pathogenic bacteria. Аnnu Rev Microbiol 37, 603-622.

Polissi, A., Pontiggia, A., Feger, G., Altieri, M., Mottl, H., Ferrari, L. \& Simon, D. (1998). Large-scale identification of virulence genes from Streptococcus pneumoniae. Infect Immun 66, 5620-5629.

Poulsen, K., Reinholdt, J. \& Kilian, M. (1996). Characterization of the Streptococcus pneumoniae immunoglobulin A1 protease gene (iga) and its translation product. Infect Immun 64, 3957-3966.

Poulsen, K., Reinholdt, J., Jespersgaard, C., Boye, K., Brown, T. A., Hauge, M. \& Kilian, M. (1998). A comprehensive genetic study of streptococcal immunoglobulin A1 proteases: evidence for recombination within and between species. Infect Immun 66, 181-190.

Reinholdt, J. \& Kilian, M. (1997). Comparative analysis of immunoglobulin Al protease activity among bacteria representing different genera, species, and strains. Infect Immun 65, 4452-4459. 
Reyn, A. (1986). Genus Gemella Berger 1960, 253AL. In Bergey's Manual of Systematic Bacteriology, vol. 2, pp. 1081-1082. Edited by P. H. A. Sneath, N. S. Mair, M. E. Sharpe \& J. G. Holt. Baltimore: Williams \& Wilkins.

Sambrook, J., Fritsch, E. F. \& Maniatis, T. (1989). Molecular Cloning: a Laboratory Manual, 2nd edn. Cold Spring Harbor, NY: Cold Spring Harbor Laboratory.

Sutcliffe, I. C. \& Harrington, D. J. (2002). Pattern searches for the identification of putative lipoprotein genes in Gram-positive bacterial genomes. Microbiology 148, 2065-2077.

Tettelin, H., Nelson, K. E., Paulsen, I. T. \& 36 other authors (2001). Complete genome sequence of a virulent isolate of Streptococcus pneumoniae. Science 293, 498-506.
Vallee, B. L. \& Auld, D. S. (1990). Active-site zinc ligands and activated $\mathrm{H}_{2} \mathrm{O}$ of zinc enzymes. Proc Natl Acad Sci U S A 87, 220-224.

von Heijne, G. (1989). Control of topology and mode of assembly of a polytopic membrane protein by positively charged residues. Nature 341, 456-458.

Wani, J. H., Gilbert, J. V., Plaut, A. G. \& Weiser, J. N. (1996). Identification, cloning, and sequencing of the immunoglobulin A1 protease gene of Streptococcus pneumoniae. Infect Immun 64, 3967-3974.

Weiser, J. N., Bae, D., Fasching, C., Scamurra, R. W., Ratner, A. J. \& Janoff, E. N. (2003). Antibody-enhanced pneumococcal adherence requires IgAl protease. Proc Natl Acad Sci U S A 100, 4215-4220. 